\title{
Uusioersily
}

\section{D printed high-performance flexible strain sensors based on carbon nanotube and graphene nanoplatelet filled polymer composites}

Xiang, D., Zhang, X., Han, Z., Zhang, Z., Zhou, Z., Harkin-Jones, E., Zhang, J., Luo, X., Wang, P., Zhao, C., \& $\mathrm{Li}, \mathrm{Y}$. (2020). 3D printed high-performance flexible strain sensors based on carbon nanotube and graphene nanoplatelet filled polymer composites. Journal of Materials Science, 55(33), 15769-15786. https://doi.org/10.1007/s10853-020-05137-w

Link to publication record in Ulster University Research Portal

Published in:

Journal of Materials Science

Publication Status:

Published (in print/issue): 01/11/2020

DOI:

10.1007/s10853-020-05137-w

\section{Document Version}

Author Accepted version

\section{General rights}

Copyright for the publications made accessible via Ulster University's Research Portal is retained by the author(s) and / or other copyright owners and it is a condition of accessing these publications that users recognise and abide by the legal requirements associated with these rights.

\section{Take down policy}

The Research Portal is Ulster University's institutional repository that provides access to Ulster's research outputs. Every effort has been made to ensure that content in the Research Portal does not infringe any person's rights, or applicable UK laws. If you discover content in the Research Portal that you believe breaches copyright or violates any law, please contact pure-support@ulster.ac.uk. 


\section{Journal of Materials Science}

\section{D Printed High-Performance Flexible Strain Sensors Based on Carbon Nanotube and Graphene Nanoplatelet Filled Polymer Composites \\ --Manuscript Draft--}

Manuscript Number:

Full Title:

Article Type:

Keywords:

Corresponding Author:
JMSC-D-20-02144R1

3D Printed High-Performance Flexible Strain Sensors Based on Carbon Nanotube and Graphene Nanoplatelet Filled Polymer Composites

Manuscript (Regular Article)

carbon nanotubes; graphene nanoplatelets; polymer composites; Sensor; 3D Printing

Dong Xiang

Southwest Petroleum University

Chengdu, Sichuan CHINA

\section{Corresponding Author Secondary}

Information:

Corresponding Author's Institution:

Southwest Petroleum University

\section{Corresponding Author's Secondary} Institution:

First Author:

Dong Xiang

First Author Secondary Information:

Order of Authors:

\begin{tabular}{|l|} 
Dong Xiang \\
Xuezhong Zhang \\
\hline
\end{tabular}

Zhuohang Han

Zixi Zhang

Zuoxin Zhou

Eileen Harkin-Jones

Jie Zhang

Xia Luo

Ping Wang

Chunxia Zhao

Yuntao Li

Order of Authors Secondary Information:

Abstract:

Funding Information:
In this study, high-performance flexible strain sensors based on carbon nanotube (CNT) and graphene nanoplatelet (GNP) filled thermoplastic polyurethane (TPU) composites were fabricated via Fused Filament Fabrication (FFF) 3D printing. The introduction of GNPs generated a more complete conductive network of the composites due to the improved nanofiller dispersion. Due to the synergy of CNTs and GNPs, the printed CNT/GNP(3:1)/TPU sensor shows higher sensitivity (GF = 136327.4 at $250 \%$ strain), larger detectable range (0 250\% strain), and better stability (3000 cycles) compared with the CNT/TPU and GNP/TPU sensors with a nanofiller content of $2 \mathrm{wt} \%$. Furthermore, the printed sensors can accurately detect strains at different frequencies $(0.01 \sim 1 \mathrm{~Hz})$. A modelling study based on tunneling theory was conducted to analysis the strain sensing mechanism, and the theoretical results agreed well with the experimental data. The capability of the sensors in monitoring physiological activities and speech recognition has also been demonstrated.
Sichuan Science and Technology Program
Dr. Dong Xiang 
(2017HH0086, 2017JY0152)

Sichuan Provincial University Key

Laboratory of Oil and Gas Field Materials

Dr. Dong Xiang

(No. X151519KCL05)

Scientific Research Foundation for

Returned Scholars of Ministry of

Dr. Dong Xiang

Education 


\section{D Printed High-Performance Flexible Strain Sensors Based on Carbon Nanotube and Graphene Nanoplatelet Filled Polymer Composites}

Dong Xianga,,*\#, Xuezhong Zhang, ${ }^{\mathrm{a}, \#}$, Zhuohang Han ${ }^{\mathrm{a}}$, Zixi Zhang ${ }^{\mathrm{a}}$, Zuoxin Zhou ${ }^{\mathrm{b}}$, Eileen Harkin-Jones ${ }^{\mathrm{c}}$, Jie Zhang ${ }^{\mathrm{d}}$, Xia Luo ${ }^{\mathrm{a}}$, Ping Wang ${ }^{\mathrm{a}}$, Chunxia Zhao ${ }^{\text {a }}$, Yuntao Lia, ${ }^{\text {,* }}$

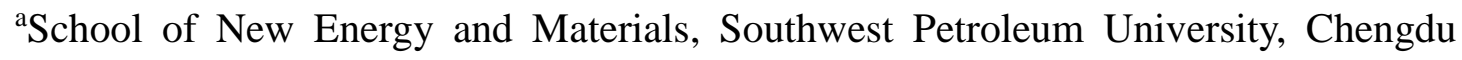
610500, China

${ }^{\mathrm{b} C e n t e r}$ for Additive Manufacturing, Faculty of Engineering, University of Nottingham, Nottingham NG7 2RD, UK

${ }^{\mathrm{c}}$ School of Engineering, University of Ulster, Jordanstown BT37 0QB, UK

${ }^{\mathrm{d} S}$ School of Mechatronic Engineering, Southwest Petroleum University, Chengdu 610500, China

*Corresponding authors: dxiang01@hotmail.com (Dong Xiang); yuntaoli@swpu.edu.cn (Yuntao Li)

\#These authors contributed equally to this work. 
ABSTRACT: In this study, high-performance flexible strain sensors based on carbon nanotube (CNT) and graphene nanoplatelet (GNP) filled thermoplastic polyurethane (TPU) composites were fabricated via Fused Filament Fabrication (FFF) 3D printing. The introduction of GNPs generated a more complete conductive network of the composites due to the improved nanofiller dispersion. Due to the synergy of CNTs and GNPs, the printed CNT/GNP(3:1)/TPU sensor shows higher sensitivity $(\mathrm{GF}=$ 136327.4 at $250 \%$ strain), larger detectable range (0 250\% strain), and better stability (3000 cycles) compared with the CNT/TPU and GNP/TPU sensors with a nanofiller content of $2 \mathrm{wt} \%$. Furthermore, the printed sensors can accurately detect strains at different frequencies $(0.01 \sim 1 \mathrm{~Hz})$. A modelling study based on tunneling theory was conducted to analysis the strain sensing mechanism, and the theoretical results agreed well with the experimental data. The capability of the sensors in monitoring physiological activities and speech recognition has also been demonstrated.

Keywords: carbon nanotubes, graphene nanoplatelets, polymer composites, sensor, 3D printing

\section{Introduction}

With the increasing level of automation in industrial production, the global market for strain sensors is expanding [1]. However, conventional strain sensors made of metal and semiconductor materials usually show limited sensitivity [2]. Also, the flexibility and strain range of such sensors do not meet the performance requirements 
of a high strain field. Therefore, there is a need to develop suitable flexible strain sensors to meet industrial needs. The rapid growth and development of nanomaterials in the last decade has enabled their use in strain sensing applications. Currently, the nanomaterials widely used in the preparation of flexible sensors include graphene nanoplatelets (GNPs) [3], reduced graphene oxide ( $\mathrm{rGO}$ ), multiwalled carbon nanotubes (MWCNTs) [4], silver nanowires (AgNWs), and silver nanoparticles (AgNPs). For instance, Zhang et al. [5] reported a method for preparing strain sensors of TPU/CNT composite films using solution blending, which reduced the percolation threshold $\left(P_{c} \approx 0.35 \mathrm{wt} \%\right)$ of CNTs, and also induced a high repeatability during the cyclic stretch-release testing of the composites. Wang et al. [6] used electrospinning method to prepare a graphene/TPU fiber sensor with a high sensitivity (gauge factor (GF) is 11 at the strain of 10\%) and good stability. However, the use of a single nanofiller in strain sensor applications has some limitations which are mainly due to filler dispersibility and optimum netwerk formanter the use of a single nanofiller in the preparation of strain sensors has some limitations which are mainly due to the poor nanofiller dispersibility and more difficulties in conductive network formation $[2,4]$. For example, one-dimensional MWCNTs are easily entangled, which may increase the strain range of the sensor but also decrease its sensitivity. Two dimensional GNPs that can slip under tension, provide high sensitivity but a low strain range [7].

Up to now, the synergistic effect of different nanofillers has been reported in much literature to improve the dispersion of nanofillers and generate more conductive 
paths [8]. Ma et al. [9] fabricated versatile piezoresistive sensors based on conductive polyurethane (PU) sponges using dip-coating layer-by-layer electrostatic assembly. The resultant conductive sponges exhibited an excellent conductivity and compressibility (up to $75 \%$ ) due to the synergistic effect of conductive $\mathrm{CNT} / \mathrm{rGO}$ structures. Peng et al. [10] fabricated a lightweight and high-performance CNF-rGO/CNT carbon aerogel using freeze drying. The synergistic effect of CNTs and cellulose nanofibers (CNFs) endowed the carbon aerogel with a high sensitivity and ultrahigh compressibility (up to $95 \%$ strain). Zhao et al. [11] prepared highly conductive multifunctional $\mathrm{rGO} / \mathrm{CNT}$ hybrid sponge-based strain sensor through chemical vapor deposition (CVD). Compared with the CNT sponge, the sensitivity of the $\mathrm{rGO} / \mathrm{CNT}$ hybrid sponge is $50 \%$ higher.

However, the preparation methods mentioned above are usually expensive and complicateddifficult to seale up for industrial use. Additionally, they are not suitable for customizing strain sensor performance due to their limited capability in structural design and control. Additive Manufacturing (AM) or 3D printing technologies fabricate objects based on digital model files via a layer-by-layer method and from a variety of materials such as powdered metals and plastic filaments and powders. Conductive polymer composites have been successfully processed using 3D printing technologies [12-19]. The techniques that have been utilized include powder bed fusion [12], vat photopolymerization [13], and fused filament fabrication [14]. Mu et al. [15] prepared a conductive polymer composite material based on MWCNTs and photocurable resin using vat photopolymerization. Li et al. [16] processed a 
CNT/TPU composite with a low percolation threshold of $0.2 \mathrm{wt} \%$ using powder bed fusion. Odent et al. [17] printed flexible and highly-conductive poly(vinylidene fluoride) (PVDF) composites containing multi-walled carbon nanotube (MWCNT) using FFF. Benefiting from its low cost and flexible structural design, 3D printing is therefore a promising processing technology for sensor manufacture. Christ et al. [18] used FFF to fabricate a flexible strain sensor based on CNT/TPU nanocomposites. The results showed that increases in CNT content improved the printability of TPU. Xiang et al. [14] reported a method to enhance the performance of FFF 3D printed strain sensors by non-covalently modifying CNTs to improve the interfacial interactions with polymer matrix. Huang et al. [19] printed carbon fiber-filled conductive silicon rubbers (CSRs) through an extruder. The printed CSRs exhibited improved mechanical and electrical properties along the alignment direction of the fibers. The printed strain sensor was capable of recognizing the bending of fingers, demonstrating its potential for monitoring human movement. Although research in this important area is increasing, the focus tends to be on optimization the structure of the 3D printed sensors and the effects of nanofiller type and composition on the performance of the printed composites is given less attention. Recently, the authors synthesized silver nanoparticles (AgNPs) by electrophoretic deposition in the presence of CNTs, then printed a highly elastic strain sensor containing the hybrid nanofillers via FFF. It was found that the sensing property of the sensor was improved with the addition of AgNPs, while the synthesis process of AgNPs was complicated and the sensitivity of sensor should be further increased [20]. 
In this work, flexible strain sensors based on CNT/TPU, GNP/TPU, and CNT/GNP/TPU nanocomposites were fabricated via the FFF 3D printing process. The dispersion of nanofillers in the TPU and the printability of the nanocomposites were studied. Furthermore, the effects of nanofiller type and synergy of hybrid nanofillers on the electrical, tensile and strain sensing properties of the 3D printed sensors were systematically researched. To understand the mechanism of strain sensing, a modelling analysis based on tunneling theory was performed. The ability of strain sensors to monitor limb motions, physiological activities, and speech recognition have also been demonstrated. This work shows an effective method for the 3D printing of high-performance flexible strain sensors with potential applications in software robots, smart wearable devices, and medical monitoring equipment.

\section{Experimental Section}

\section{Materials}

Multi-walled carbon nanotubes (NC7000) with a length and a diameter of $1.5 \mu \mathrm{m}$ and $9.5 \mathrm{~nm}$, respectively, were sourced from the Nanocyl (Belgium). Graphene nanoplatelets with an average of 5 6 layers were provided by the Carbonene Co. Ltd (China). The thickness and diameter of the GNPs are of $3 \mathrm{~nm}$ and $5 \sim 15 \mu \mathrm{m}$, respectively. Thermoplastic polyurethane (TPU, Bayer 2195) particles, with a mass fraction of hard segment of $36 \mathrm{wt} \%$, a density of $1.19 \mathrm{~g} / \mathrm{cm}^{3}$ and a melt flow index of $12.1 \mathrm{~g} / 10 \mathrm{~min}\left(210^{\circ} \mathrm{C}\right.$, at a pressure of $\left.5 \mathrm{~kg}\right)$ was purchased from the Bayer Co. Ltd. Dimethylformamide (DMF) reagent was supplied by the Chron Chemicals Co. Ltd 
(China).

\section{Preparation of nanocomposites}

Nanocomposites with $0.5 \sim 5 \mathrm{wt} \%$ nanofiller loadings were prepared. CNTs and GNPs at different weight ratios were dispersed in DMF by ultra-sonicating at $100 \mathrm{~W}$ for $1 \mathrm{~h}$. Then, the TPU particles were introduced into the DMF solvent and the mixture was magnetically stirring for $2 \mathrm{~h}$ for a complete dissolution. The mixed suspension was flocculated with absolute ethanol to remove the less volatile DMF. The flocculated product was placed in a forced air oven at $80{ }^{\circ} \mathrm{C}$ for $24 \mathrm{~h}$ to remove the remaining solvent and to obtain nanocomposite sheets (Figure 1). The masses of raw materials used to prepare CNT/GNP/TPU composites with various CNTs:GNPs weight ratios (7:1, 3:1, 1:1, 1:3, and 1:7) are listed in Table 1 for a total content of 2 wt\% nanofillers. CNT/TPU and GNP/TPU composites were also prepared by the same method and used as references.

Table 1. Content of raw materials for preparing CNT/TPU, GNP/TPU, and

\begin{tabular}{ccccc}
\hline Sample & Nanofiller ratio & CNTs & GNPs & TPU \\
& $(\mathrm{CNT}$ : GNP $)$ & $(\mathrm{g})$ & $(\mathrm{g})$ & $(\mathrm{g})$ \\
\hline CNT/TPU & - & 0.40 & - & 19.60 \\
$\mathrm{CNT/GNP}(7: 1) / \mathrm{TPU}$ & $7: 1$ & 0.35 & 0.05 & 19.60 \\
$\mathrm{CNT/GNP}(3: 1) / \mathrm{TPU}$ & $3: 1$ & 0.30 & 0.10 & 19.60 \\
$\mathrm{CNT/GNP}(1: 1) / \mathrm{TPU}$ & $1: 1$ & 0.20 & 0.20 & 19.60 \\
\hline
\end{tabular}




\section{FFF 3D printing of nanocomposites}

The prepared composite material was added to a desktop single-screw extruder (Wellzoom Type C) to produce a composite feedstock filament with a diameter of $1.75 \mathrm{~mm}$. The processing temperature of the extruder was $210{ }^{\circ} \mathrm{C}$, and the screw speed was $100 \mathrm{rev} / \mathrm{min}$. The filaments were then processed via FFF using an ET-K1 desktop 3D printer (ET Co. Ltd., China). A stacking mode with an interlayer angle of $90^{\circ}$ was applied (Figure 1). The nozzle temperature was set at $220^{\circ} \mathrm{C}$ to fully melt the composite filament (Table 2). Filament was deposited at $20 \mathrm{~mm} / \mathrm{s}$ onto a substrate that was maintained at $70{ }^{\circ} \mathrm{C}$ to allow better adhesion of the first layer deposited. $100 \%$ infill was employed. The layer thickness was $0.1 \mathrm{~mm}$. The dimension of the printed samples was $50 \mathrm{~mm} \times 10 \mathrm{~mm} \times 1 \mathrm{~mm}$. 


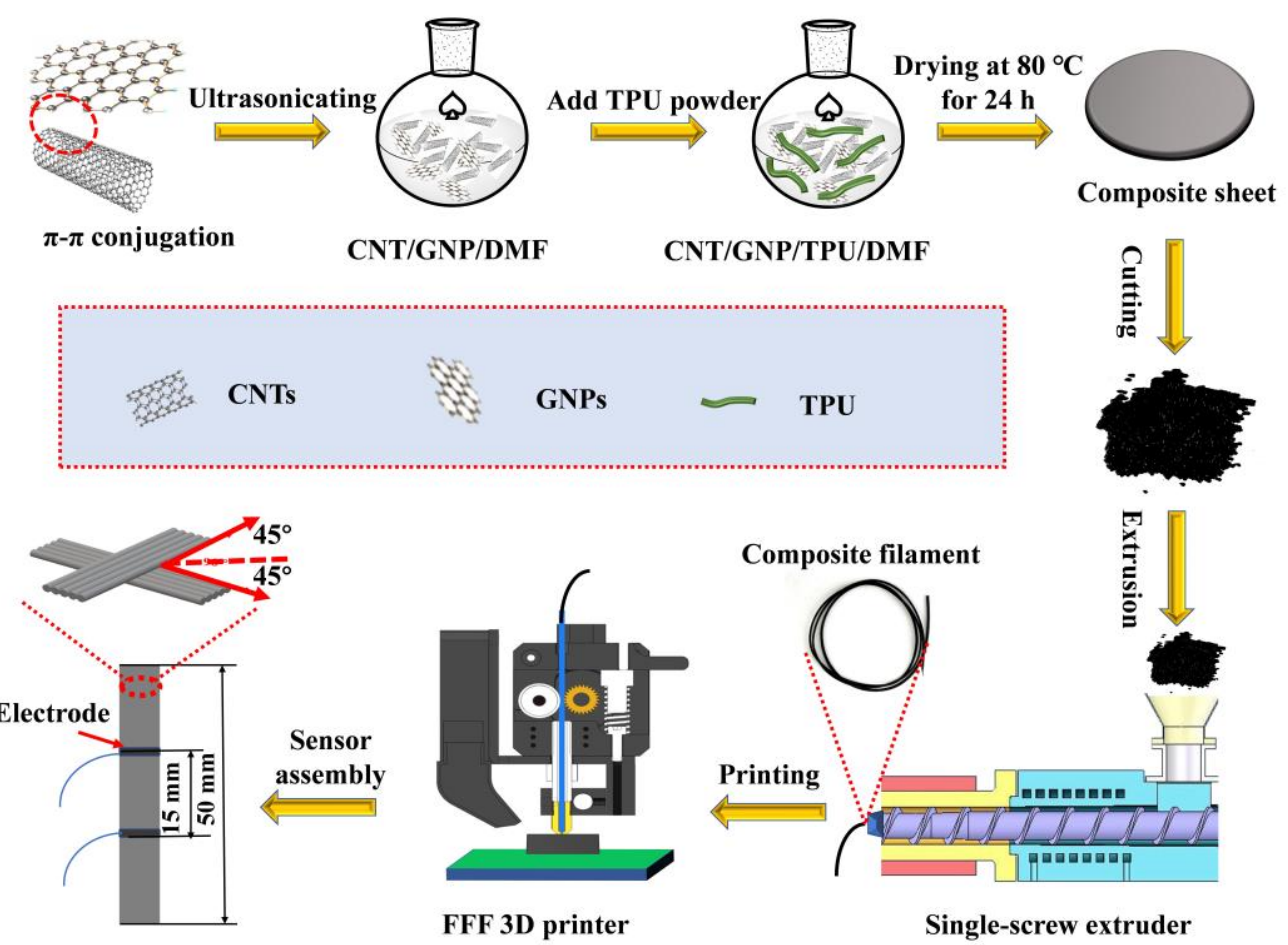

Figure 1 Schematic diagram of the 3D printing of the flexible strain sensor.

Table 2. 3D printing parameters for the nanocomposite strain sensors.

\begin{tabular}{cl}
\hline Parameter & Value \\
\hline Layer thickness $(\mathrm{mm})$ & 0.1 \\
Infill $(\%)$ & 100 \\
Printing speed $(\mathrm{mm} / \mathrm{s})$ & 20 \\
Hot bed temperature $\left({ }^{\circ} \mathrm{C}\right)$ & 70 \\
Nozzle temperature $\left({ }^{\circ} \mathrm{C}\right)$ & 220 \\
Nozzle diameter $(\mathrm{mm})$ & 0.4 \\
\hline
\end{tabular}

\section{Characterization}

Zeta potentiometer (Brookhaven Zeta PALS 190 Plus) was used to analyze the dispersibility of CNTs, GNPs and CNT/GNP nanofillers in DMF. UV-Vis spectra 
were recorded on a Perkin EImer Lambda 850 to help to verify the dispersion of the suspensions. X-ray diffraction (XRD) was conducted on a D8-Adcance instrument (Germany) and using $\mathrm{Cu}-\mathrm{K} \alpha$ radiation. The scanning was conducted at a speed of $0.02^{\circ} \mathrm{min}^{-1}$ from 10 to $50^{\circ}$. Field emission scanning electron microscopy (FESEM) images were obtained using a FEI Quanta 650 FEG apparatus under an accelerating voltage of $30 \mathrm{kV}$. All the samples were not sprayed with gold in order to enable observation of the conductive network via the secondary electrons emitted from the conductive nanofillers. An MTS CMT4104 Universal Tester was used to characterize the tensile properties of samples. The conductivity of the nanocomposites was measured using a two-point method in combination with a picoamp-meter (Keithley 6485 ) and a DC digital source meter (Tektronix PWS4323) at a voltage of $3 \mathrm{~V}$. The electrode distance was $15 \mathrm{~mm}$. Silver paste was used to minimize the contact resistance between sample and electrode. The sensing performance of the strain sensor was also tested using picoamp-meter, DC digital source meter and universal tester mentioned above.

\section{Results and Discussion}

\section{Dispersion of nanofillers}

The CNT/DMF, GNP/DMF, and CNT/GNP(3:1)/DMF suspensions (200 mg/100 $\mathrm{mL}$ ), were left standing for one week after $1 \mathrm{~h}$ ultrasonication. The zeta potentials $(\zeta)$ of the nanofiller/DMF suspensions are displayed in Fig. 2a. The $\zeta$ potential of the CNT/GNP(3:1)/DMF suspension before standing was $38.6 \mathrm{mV}$, which is higher than 
that of CNT/DMF suspension $(27.2 \mathrm{mV})$. After one week of standing, the $\zeta$ potential of the nanofiller/DMF suspensions decreased to different extents. The CNT/GNP(3:1)/DMF suspension exhibited the smallest decrase in $\zeta$ (from 38.6 to $36.3 \mathrm{mV}$ ) after standing for one week, indicating that GNPs have a positive impact on improving the dispersion of CNTs due to their large specific surface area and ability to separate the CNTs spatially. Fig. 2b shows the UV-Vis spectra of all samples before and after one week standing. The spectrum of the nanofiller/DMF suspension shows a distinct absorption peak at $255 \mathrm{~nm}$ due to the transition of the $\pi-\pi$ conjugated electrons of the nanofiller. A higher absorption peak indicates a better dispersion of the nanofillers in the suspension. The UV absorption peaks of the CNT/GNP(3:1)/DMF suspensions before and after standing are higher than those of the CNT/DMF and GNP/DMF suspensions, indicating that the CNT and CNP hybrid nanofillers exhibit better dispersion and stability. Fig. 2c exhibits the photographs of the nanofiller/DMF suspensions before and after standing for one week. It can be seen that most of the CNTs have precipitated to the bottom of the bottle after standing. However, due to the polar groups left on the surface of GNPs, the suspension made with GNPs maintained a good homogeneous state.

Fig. 2d shows the XRD spectra of CNTs, GNPs, neat TPU, CNT/TPU, GNP/TPU, and CNT/GNP/TPU nanocomposites with 2 wt\% nanofiller. The diffractogram of the CNTs has two diffraction peaks at around $25.1 z^{\circ}(002)$ and $43.2^{\circ}$ (100), which are attributed to the in-plane graphitic structure and the interlayer space in the radial direction of CNTs, respectively [21]. The weak and broad diffraction 
peak belonging to GNPs appears about $25.1^{\circ}$, which can be assigned to the (002) planes of the graphite structure with short-range ordered structure in GNPs. The XRD pattern of neat TPU shows a wide diffraction peak at $20.1^{\circ}$, which denotes a short-range, ordered structure of both soft and hard domains along with a disordered structure of the amorphous phase of the TPU matrix [22]. The XRD pattern of CNT/TPU, has the diffraction peak of TPU at $20.1^{\circ}$, but also the diffraction peak of CNTs at $25.21^{\circ}$. It suggests that CNTs are poorly dispersed in the TPU matrix. The diffractograms of GNP/TPU and CNT/GNP(3:1)/TPU have only the diffraction peak of TPU, suggesting that GNPs and CNT/GNP hybrid nanofillers are well dispersed in the TPU matrix. Table S1 (Supporting Information) shows that the crystallinity $\left(X_{c}\right)$ of TPU decreases with the addition of nanofillers due to nanofiller agglomerates reducing the mobility of the TPU chains. The $X_{c}$ of CNT/TPU and CNT/GNP(3:1)/TPU composites decreased by $42 \%$ and $16 \%$, respectively, compared with neat TPU. This also confirms that the addition of GNPs significantly facilitates the dispersion of nanotubes in the matrix. 
(a)

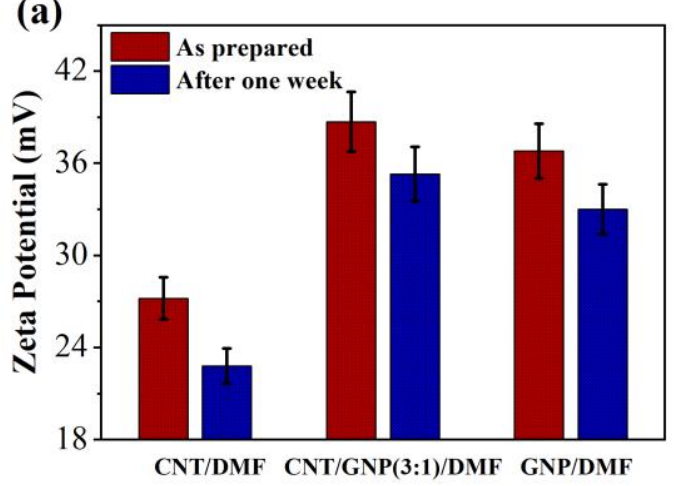

(c)

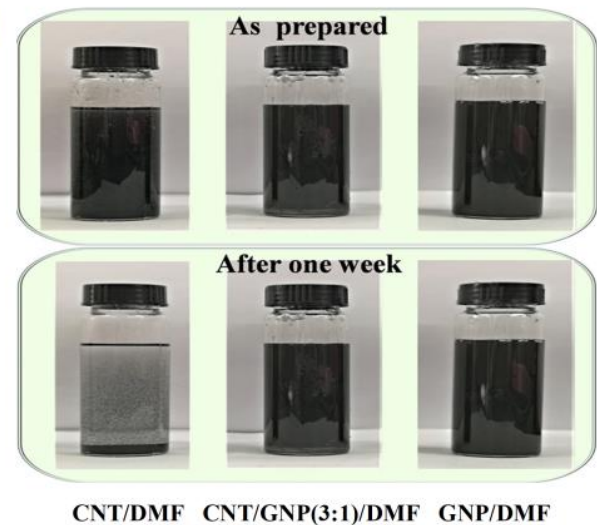

(b)

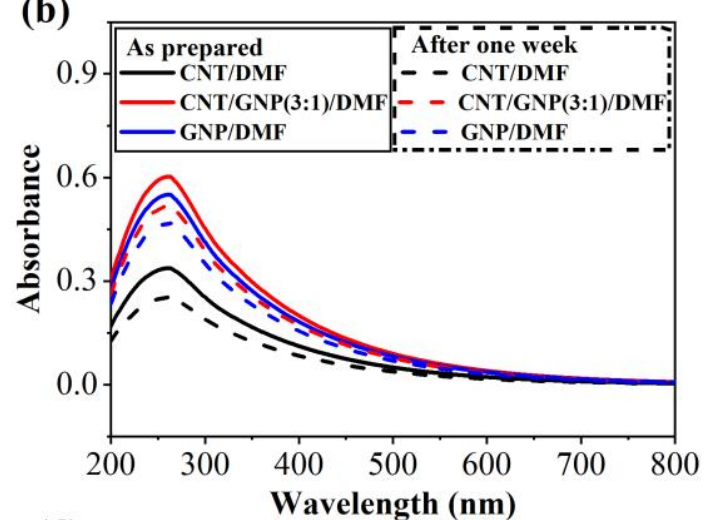

(d)

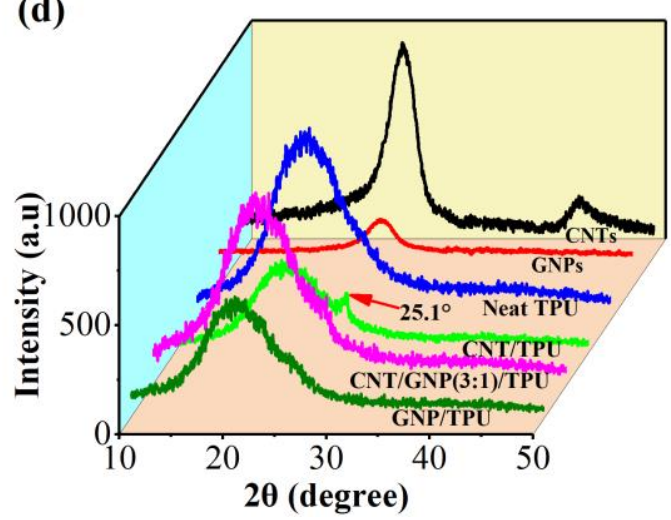

Figure 2 (a) Zeta potential ( $\zeta$ ) of the nanofiller/DMF suspension before and after standing for one week, (b) UV-Vis spectra of nanofiller/DMF suspensions before and after standing for one week, (c) Digital photos of nanofiller/DMF suspensions before and after standing for one week, (d) XRD patterns of the neat TPU, CNT/TPU, GNP/TPU, and CNT/GNP(3:1)/TPU samples.

\section{Printability and morphology}

If the filament is not sufficiently rigid it will buckle at the entrance to the extruder of the FFF device and prevent extrusion of the filament through the printer nozzle. The critical buckling pressure $\left(P_{c r}\right)$ of the filament can be calculated using the Euler formula [23]:

$$
P_{c r}=\frac{\pi^{2} E D_{f}^{2}}{16 L_{f}^{2}}
$$


where $D_{f}$ is the diameter of the nanocomposite filament, $E$ is the elastic modulus of the filament, and $L_{f}$ is the length of filament from the melting zone to the drive gear.

Fig. $3 \mathrm{a}$ and $\mathrm{b}$ present the curves of the elastic modulus and buckling pressure, respectively. It can be observed that the $E$ and $P_{c r}$ of the filament increase almost linearly with the increase in nanofiller content. When the weight fraction of CNTs is 2 wt $\%, E$ and $P_{c r}$ of the CNT/TPU filaments are $19.8 \mathrm{MPa}$ and $7.6 \mathrm{kPa}$, respectively. For a weight fraction of CNTs of $5 \mathrm{wt} \%$, the values of $E$ and $P_{c r}$ of the CNT/TPU filaments are $26.9 \mathrm{MPa}$ and $1.03 \mathrm{kPa}$, respectively. Hence, the $P_{c r}$ values for 2 and 5 wt $\%$ CNT/TPU filaments increase by 17.1 and $59.1 \%$, respectively, compared with that of neat TPU filament. However, excessive filler content causes agglomeration of the nanofillers in the polymer matrix, which is detrimental for the printability of the nanocomposites. When the content of the nanofiller is $2 \mathrm{wt} \%$, the printability of the composites changes with the weight fraction ratios of CNTs and GNPs as shown in Fig. $3 c$ and d. $E$ and $P_{c r}$ values of the of GNP/TPU filaments are slightly higher compared with those of CNT/TPU filaments, indicating that the enhancement effect of the two-dimensional GNPs is better than that of one-dimensional CNTs. Interestingly, at the same loading degree of nanofiller (2 wt $\%$, the CNT/GNP(3:1)/TPU filament displays the highest $E$ and $P_{c r}(21.2 \mathrm{MPa}$ and $8.2 \mathrm{kPa}$, respectively) compared with the other nanocomposite filaments. It also exhibits the best printability. However, the $E$ and $P_{c r}$ of the nanocomposite filaments decrease as the amounts of GNPs increases probably due to the formation of GNP agglomerates. 
(a)

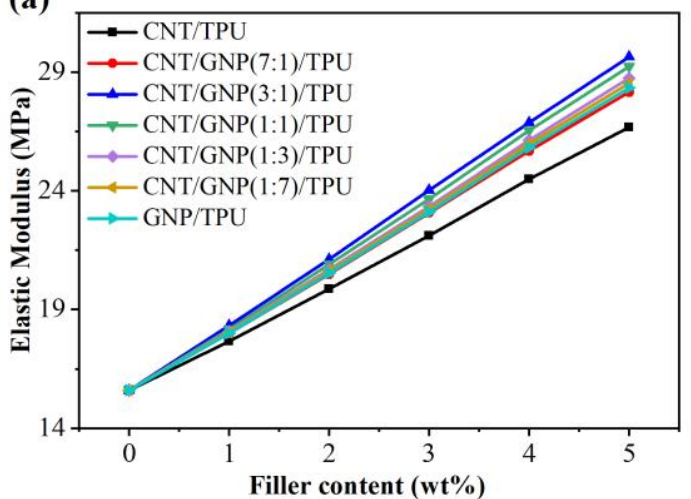

(c)

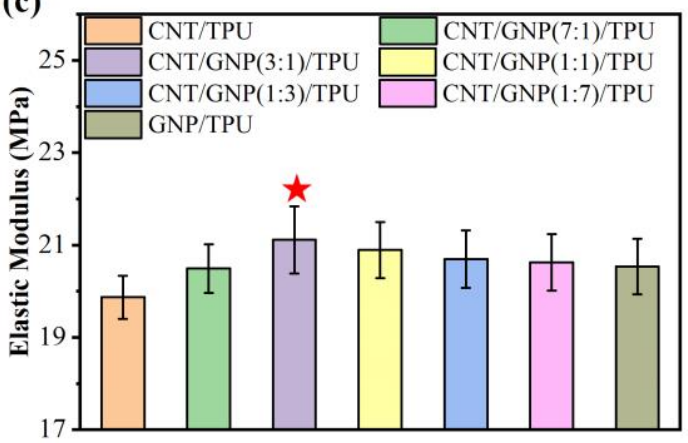

(b)

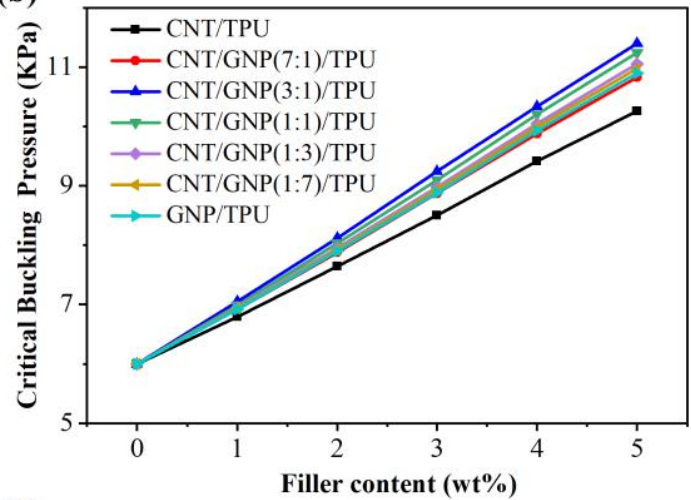

(d)

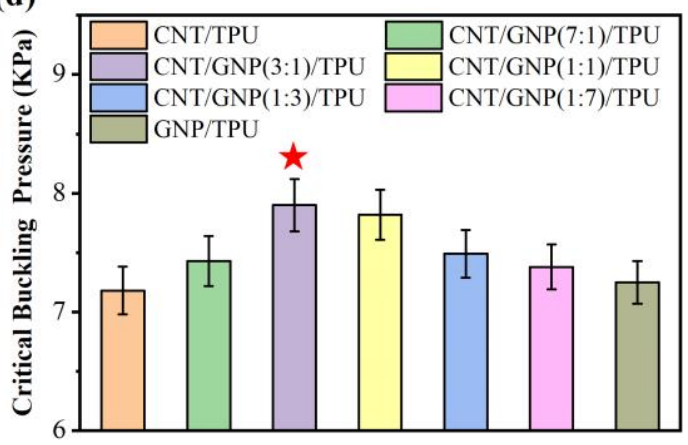

Figure 3 The curves of $E$ (a) and $P_{c r}$ (b) of the extruded filaments versus the nanofiller content, Values of $E$ (c) and $P_{c r}$ (d) of the extruded filament at different weight ratios of CNTs and GNPs.

Figure 4 shows the FESEM images of the printed composites at different magnifications. Fig. $4 \mathrm{a}$ and $\mathrm{b}$ show the surface and cross-section structures of the printed CNT/TPU composites, respectively. It can be observed that the printed samples have a distinct multi-layer structure with a layer thickness of approximately $0.1 \mathrm{~mm}$ (Fig. 4b). Yet some voids exist in the printed nanocomposites, despite the good adhesion between layers. It can be seen in Fig. 4c-d and Fig. S1a-b (Supporting information)d that numerous CNT agglomerates are distributed in CNT/TPU nanocomposites. Fig. 4e and f show the surface and cross-section morphology of the GNP/TPU composites. The edges of numerous GNPs can be clearly seen on the surface of sample due to their large diameter (Fig. 4e), while they are well embedded 
in the matrix according to the fractured cross-section morphology of sample (Fig. $4 \mathrm{f}$ and Fig. S1c-d). Compared with the CNT/TPU nanocomposites, the flake-like GNPs in the GNP/TPU nanocomposites displays a better dispersion. The morphology of GNP loaded on the surface of the printed CNT/GNP(3:1)/TPU nanocomposites is more difficult to define due to being covered by pelymer wrapped CNTs (Fig. 4g and Fig. S1e-f). As shown in Fig. 4 h, the CNTs and GNPs are more uniformly dispersed in the matrix. This result is due to the synergistic effect of combining GNPs and CNTs, which improves the dispersion of nanofillers.

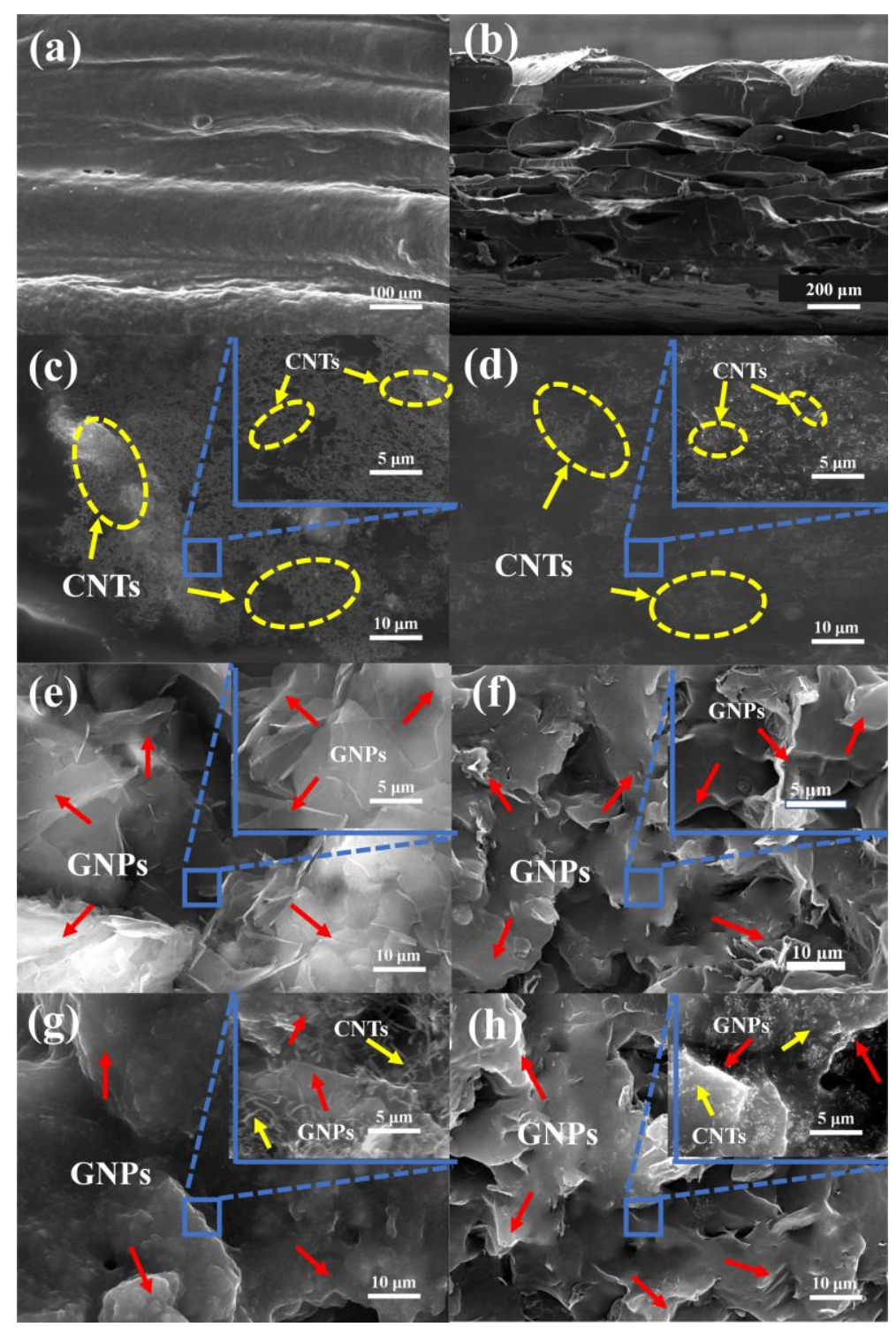


Figure 4 FESEM of the surface (left) and cross-sections (right) of printed nanocomposites with 2 wt\% nanofillers: (a-d) CNT/TPU, (e-f) GNP/TPU, (g-h) CNT/GNP(3:1)/TPU. The samples were not sprayed with gold. The CNTs and GNPs are pointed out using yellow and red arrows, respectively.

\section{Electrical properties}

The electrical volume conductivity $(\sigma)$ of the printed nanocomposites was obtained using Eq. (2):

$$
\sigma=\frac{1}{R} \times \frac{L}{S}=\frac{I}{U} \times \frac{L}{S}
$$

where $R$ is the electrical volume resistance of the sample, $U$ and $I$ are the voltage and current applied on the sample, respectively, $S$ and $L$ are the cross-sectional area of the sample and electrode distance, respectively [24].

The conductivity of the nanocomposite beyond the percolation threshold can be fitted using the power law [25]:

$$
\sigma \propto\left(f-f_{c}\right)^{t} \quad \text { for } f>f_{c}
$$

where $f$ and $f_{c}$ are the content of nanofillers and the critical content of nanofillers for the composites at percolation threshold, respectively and $t$ is the critical exponent of the conductive region. The formula follows a power-law dependence of about 1.0 1.3 in a two-dimensional system and 1.6 2.0 in a three-dimensional system.

Fig. 5a and $\mathrm{b}$ show the conductivity curves obtained for the samples. By applying classic percolation theory, a percolation threshold $\left(f_{c}\right)$ of $1.98 \mathrm{wt} \%$ is obtained for the CNT/TPU sample. The GNP/TPU has a lower percolation threshold 
$\left(f_{c}=1.67 \mathrm{wt} \%\right)$ than the CNT/TPU as a result of the high aspect ratio and improved dispersion of GNPs. The synergistic effect of CNTs and GNPs produces a more complete conductive network structure in the TPU, which further reduces the percolation threshold $\left(f_{c}=1.42 \mathrm{wt} \%\right)$ of the CNT/GNP(3:1)/TPU composites. The values of the critical exponents $(t)$ are in the range 1.6 to 2 for all printed nanocomposites (Fig. 5b), indicating that all samples have a three-dimensional conductive network.

The conductive network in the TPU and the synergistic effect of CNTs and GNPs are illustrated in Fig. 5e. Many agglomerates of nanotubes are distributed in the CNT/TPU composites. Nevertheless, more individual GNPs are evenly dispersed in the TPU matrix. GNPs favor the construction of a more efficient conductive network in the TPU. As a result, the GNP/TPU composite has a lower percolation threshold compared with that of the CNT/TPU nanocomposite. When the GNPs and CNTs are simultaneously introduced in the TPU matrix, the GNPs act as "spacers" that hinder the agglomeration of CNTs [8]. Furthermore, the one-dimensional CNTs bridge the gaps between GNPs, facilitating more conductive paths. Therefore, the addition of GNPs can improve the electrical conductivity of the resulting nanocomposite and reduce its percolation threshold.

The amount of GNPs is also an important factor influencing the electrical conductivity of nanocomposites (Fig. 5c). The conductivity of the CNT/TPU and GNP/TPU composites with $2 \mathrm{wt} \%$ nanofiller content is $3.51 \times 10^{-6}$ and $0.59 \times 10^{-6}$ S/cm, respectively. However, the CNT/GNP(3:1)/TPU shows the highest conductivity 
$\left(1.92 \times 10^{-5} \mathrm{~S} / \mathrm{cm}\right)$ at the same nanofiller loading. As the results show in Fig. 5e, when the content of GNPs is too small, agglomeration of CNTs can still occur in the nanocomposite, which limits the improvement of the electrical conductivity of the composite. When CNTs:GNPs = 3:1, the electrical conductivity of the nanocomposite is significantly enhanced due to the low content of agglomerates. However, further increase in the content of GNPs results in a reduction of available CNTs to bridge the gap between GNPs and thus leads to a decrease in the conductivity of the nanocomposite. In summary, CNT/GNP(3:1)/TPU exhibits better printability (Section 3.2) and electrical performance compared to other nanocomposites with the same nanofiller content. The mechanical and sensing properties of these particular nanocomposites are therefore investigated to see if this synergism extends to mechanical and sensing performance.

As is shown in Fig. 5d, the conductivity of nanocomposites is unchanged within the range of temperature from 20 to $80{ }^{\circ} \mathrm{C}$, indicating that the conductivity of the nanocomposite is independent of temperature. This behavior is essential for the application and precision of sensors at different temperatures. 

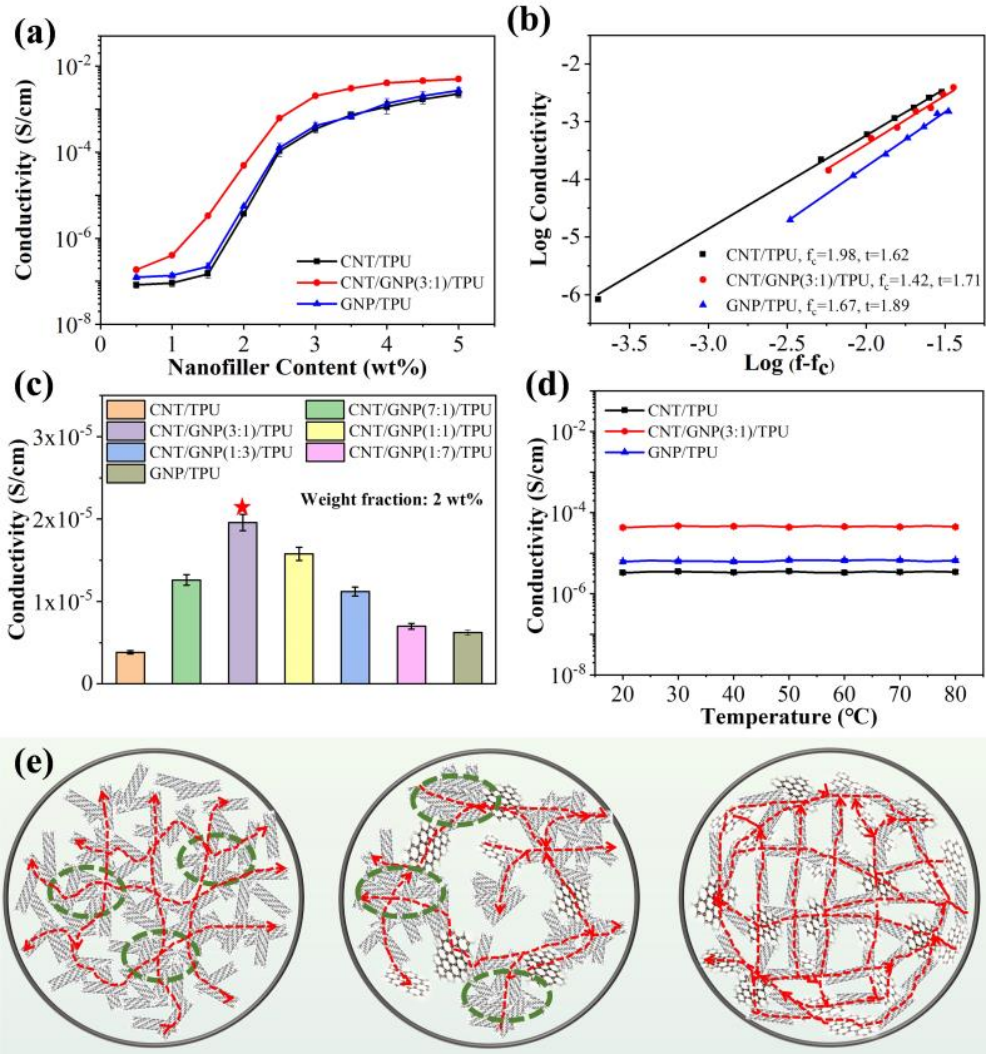

CNT/TPU
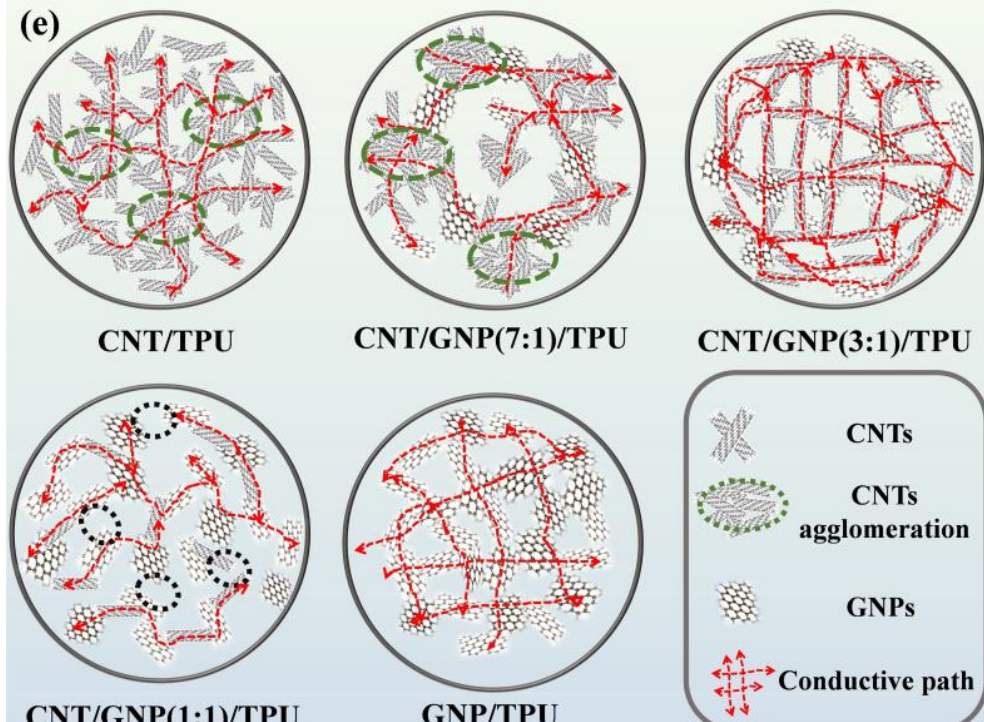

CNT/GNP(3:1)/TPU

Figure

(a) Volume

conductivity

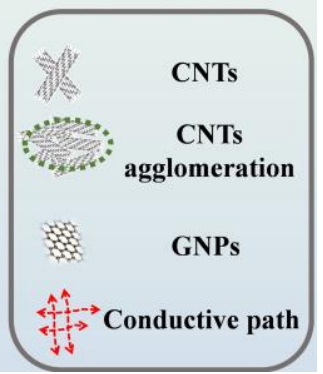

CNT/GNP(3:1)/TPU nanocomposites with different nanofiller content, (b) The curve of $\log$ conductivity versus $\log \left(f-f_{c}\right)$,

(c) Volume conductivity of CNT/TPU, GNP/TPU, and CNT/GNP/TPU nanocomposites with 2 wt $\%$ nanofiller content and different nanofiller ratios, (d) Volume conductivity versus temperature, (e) Schematic illustration of the synergistic effect of CNT and GNPs on dispersion and conductive network formation. 


\section{Tensile properties}

The tensile properties of a printed composites have a significant effect on the performance of the sensor so it is important to assess tensile performance. Fig. 6a reveals that all the printed composites show a similar strain-stress curves. Furthermore, one can see a clear strain hardening behavior when the strain is over $200 \%$. The results displayed in Fig. 6b show that, due to effective stress transfer to the high modulus nanofillers in the polymer matrix, the elastic modulus of CNT/TPU and GNP/TPU increases by 28.8 and $30.1 \%$, respectively, compared with that of neat TPU ( $E=14.5 \mathrm{MPa})$. The CNT/GNP(3:1)/TPU exhibits the highest modulus of $23.2 \mathrm{MPa}$ which is an improvement of $60.1 \%$ over the TPU modulus. The enhancement in modulus is accompanied by an expected decrease in elongation at break $\left(\varepsilon_{b}\right)$ with the largest drop being for the GNP/TPU (304.6\% versus $710.3 \%$ for the TPU, a $57.1 \%$ reduction), as shown in Fig.6c. This can be mainly attributed to the smooth surface of the two-dimensional GNPs, resulting in fracture extension along the GNP/polymer interface. The CNT/GNP(3:1)/TPU exhibits the lowest reduction in elongation $\left(\varepsilon_{b}=\right.$ $540.3 \%)$ and highest tensile strength $\left(\sigma_{b}=12.6 \mathrm{MPa}\right.$ from Fig.6d) thus preserving the synergistic effect observed in the electrical conductivity performance.

Changes in tensile properties during cyclic loading/unloading play an important role in the stability of the strain sensor. From Fig. 6e-g, it can be seen that the nanocomposites exhibit clear mechanical hysteresis due to strain softening [26] and the Mullin's effect [27] during cyclic stretching/releasing processes at large strains. The stress-strain curves of the nanocomposites show a significant change in the first 
10 cycles under a strain of $100 \%$, but they tend to be stable after that. As is shown in Fig. 6h, the mechanical hysteresis of the samples during stretching/releasing cycles is obtained by calculating the area of the curves. It can be observed that CNT/GNP(3:1)/TPU displays a smaller mechanical hysteresis than GNP/TPU and CNT/TPU nanocomposites, which is resulted from the improved dispersion of nanofillers in the TPU.
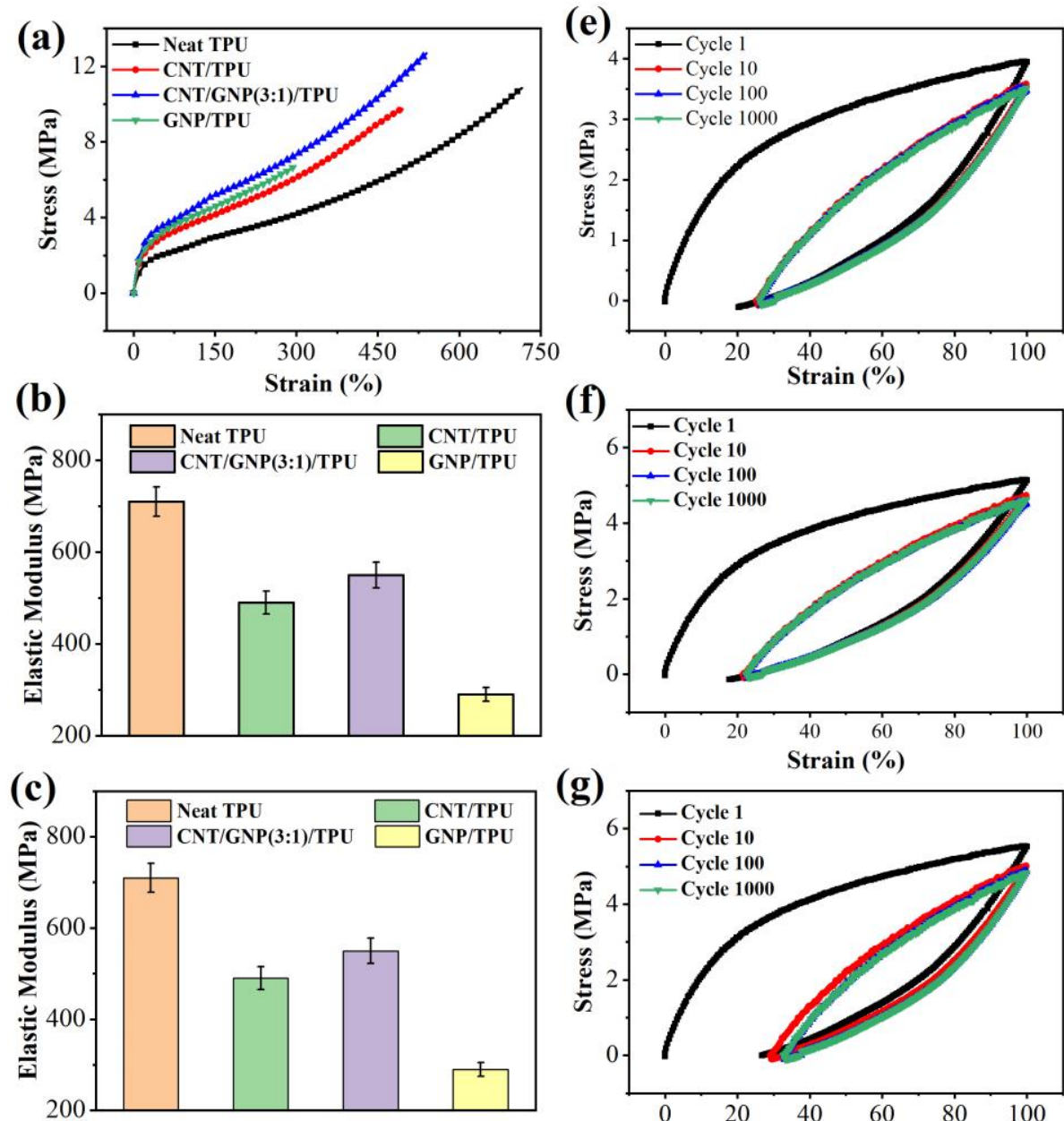

(g)
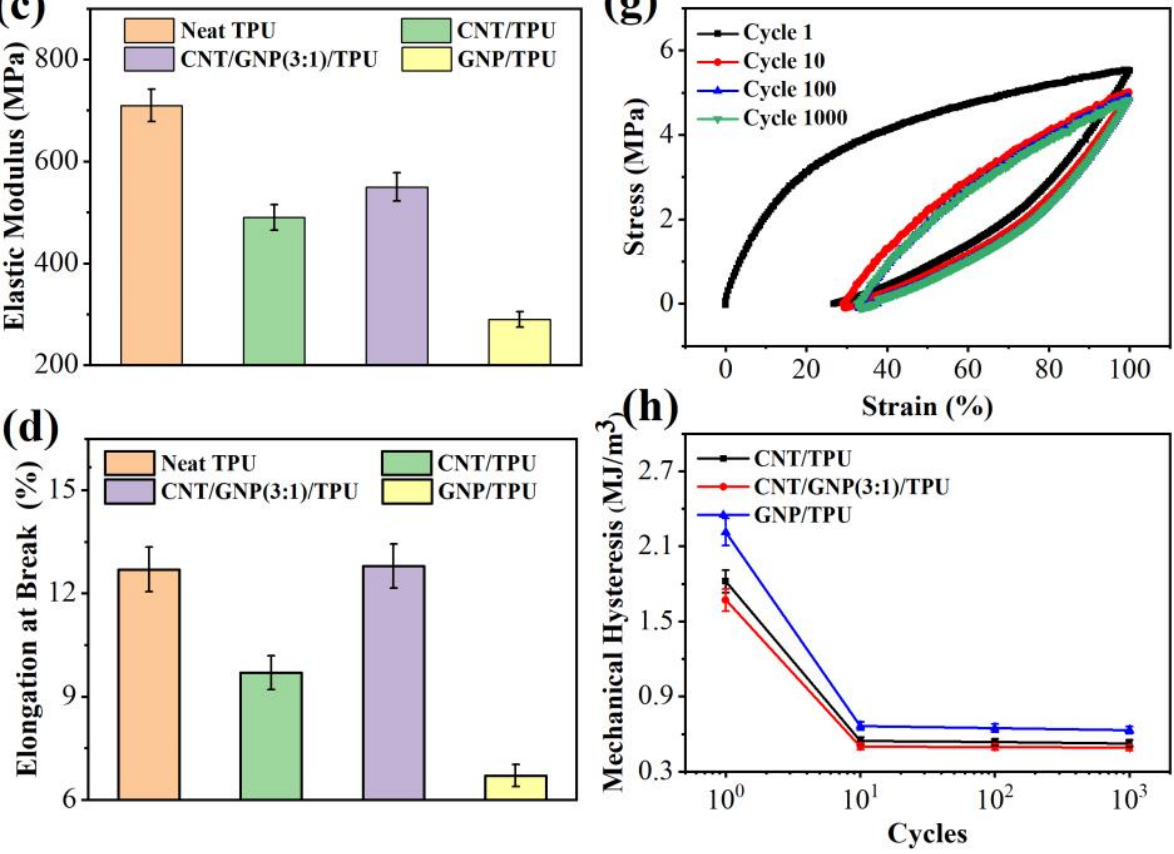
Figure 6 (a) Typical strain-stress curves, (b) elastic modulus, (c) elongation at break, and (d) tensile strength for the printed CNT/TPU, GNP/TPU, and CNT/GNP(3:1)/TPU nanocomposites with 2 wt\% nanofillers, (e-g) Stress-strain curves and (h) mechanical hysteresis of the printed nanocomposites during 1000 loading cycles at $100 \%$ strain.

\section{Electromechanical performance}

The gauge factor (GF) is often applied to quantify the sensitivity of strain sensors. GF is calculated according to Eq. (4) [28].

$$
\mathrm{GF}=\frac{\Delta R / R_{0}}{\varepsilon}
$$

where $\varepsilon, R_{0}$ and $\Delta R$ are the tensile strain, the initial resistance and the resistance change under strain, respectively [29]. It should be noted that the printed sensors for further electromechanical performance have a total nanofiller content of $2 \mathrm{wt} \%$.

Fig. 7a presents the relation between $\varepsilon$ and $\Delta R / R_{0}$ for the printed samples. The $\Delta \mathrm{R} / \mathrm{R}_{0}$ of the strain sensors gradually increases as the strain increases, indicating an obvious strain sensing behavior. Although the CNT/TPU nanocomposite exhibits an excellent strain detectable range (from 0 250\%) and linearity $\left(\mathrm{R}^{2}=0.97\right.$ at the strain of $0 \sim 30 \%$ ), its sensitivity is limited (GF $=5.67$ at the strain of 30\%). This effect is related to many CNT agglomerates in the polymer matrix. The GNP/TPU nanocomposites exhibit an outstanding sensitivity (GF $=67.31$ at $30 \%$ strain), but the strain range (from $0 \sim 125 \%)$ and linearity $\left(\mathrm{R}^{2}=0.76\right.$ at the strain of $0 \sim 30 \%$ ) are significantly lower than those of CNT/TPU nanocomposites. Since they are 
two-dimensional nanofillers, the GNPs do not generally interlace. Hence, the conductive network formed by GNPs is prone to deform and break during stretching. Still, it is observed that the CNT/GNP(3:1)/TPU nanocomposites exhibit a large strain range (from 0 250\%), excellent linearity $\left(\mathrm{R}^{2}=0.94\right.$ at the strain of $0 \sim 30 \%$ ), and high sensitivity ( $\mathrm{GF}=31.82$ at the strain of $30 \%$ ). To highlight the properties of the CNT/GNP(3:1)/TPU strain sensor demonstrated in this paper, a comparison with the properties of other stain sensors publised recenlty is illustrated in Fig. 7b [20, 30-39]. As the values show, the sensor constructed in this work shows an excellent properties in both sensitivity ( $\mathrm{GF}=136327.4$ at the strain of $250 \%)$ and detectable strain range.
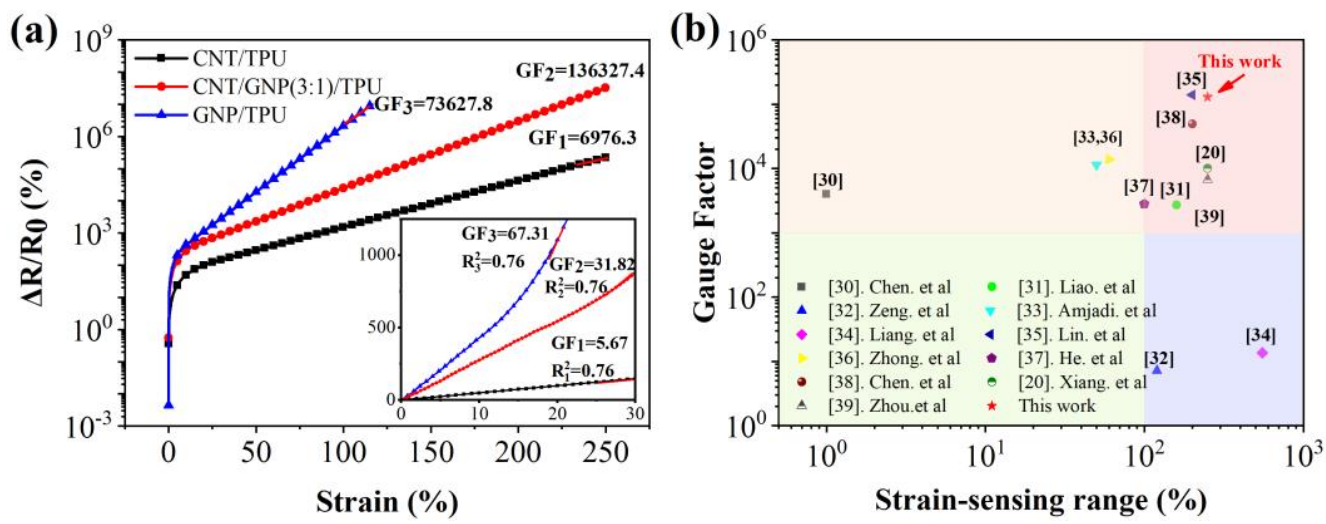

Figure 7 (a) Relation of the $\Delta R / R_{0}$ and strain for the strain sensors with different nanofillers, (b) Values of the GF and workable strain-sensing range for the CNT/GNP(3:1)/TPU strain sensor and for those recently reported in the literature.

The strain sensing behavior of printed nanocomposites was further studied under cyclic stretching/releasing conditions at various strains with a frequency $(v)$ of $0.1 \mathrm{~Hz}$. The results displayed in Fig. 8a-c reveal that the $\Delta \mathrm{R} / \mathrm{R}_{0}$ of the sensor changed consistently to the stretching/releasing cycles at strains $(\varepsilon)$ of $5,15,30$, and $50 \%$, showing the ability of the sensor to detect multiple strain deformations. From Fig. 8a, 
one can see that the $\Delta \mathrm{R} / \mathrm{R}_{0}$ of the CNT/TPU nanocomposite displays distinct "double peaks" during the cyclic stretching [31]. The "main peak" is related to the sensitivity at the maximum strain in a single loading cycle while the "shoulder peak" is caused by the competition between reconstruction and destruction of the conductive network over cyclic stretching. The mechanical hysteresis discussed above can be used to explain the phenomenon observed during cyclic stretching (Fig. 6e and h). Not all polymer chains move to their initial state after releasing [30]. This hysteresis destroys the conductive network formed by CNTs, generating more evident shoulder peaks as the strain increases [19]. These results show that the combination of CNTs and GNPs is also beneficial for good repeatability and stability of the resistance change of CNT/GNP(3:1)/TPU nanocomposites under different strains (Fig. 8b). As shown in Fig. $8 \mathrm{c}$, during the cyclic loading of the GNP/TPU nanocomposite at a large strain $(\varepsilon$ $=50 \%$ ), the resistance in the second cycle increases significantly. The likely cause of this behavior is the slippage of GNPs during stretching, which causes an irreversible deformation of the conductive network. This finding is consistent with the large mechanical hysteresis of GNP/TPU during the first cycle of cyclic stretching (Fig. $6 \mathrm{f}$ and $h)$.

The $\Delta \mathrm{R} / \mathrm{R}_{0}$ of the strain sensor at $5 \%$ strain and various frequencies $(v=0.01$, 0.1, 0.2, and $1 \mathrm{~Hz}$ ) was also studied, and the resutls are depicted in Fig. 8d-f. All the sensors show outstanding responses within the broad frequency range, highlighting the applicability of the sensor to monitor human activity at different frequencies. Notably, due to the decreased molecular mobility of polymer at high frequency, $\Delta R / R_{0}$ 
slightly increases with the increase in strain frequency.

Additionally, cyclic loading/unloading tests (up to 3000 cycles) at small (5\%) and large $(100 \%)$ strains were carried out for the printed sensors at a frequency of 1 $\mathrm{Hz}$ to investigate the repeatability and robustness of the strain sensor. The results are shown in Fig. 8g-1. Fig. 8g-i show that each sensor exhibits good stability during the 3000 loading/unloading cycles at a small strain of 5\%. The resistance of the CNT/TPU and GNP/TPU composites increased significantly during the 3000 loading/unloading cycles at $100 \%$ strain, emphazing their relatively low stability and repeatability (Fig. 8j and 1). However, from Fig. 8k, it is clear that the CNT/GNP(3:1)/TPU composites exhibits superior stability and more uniform signal responses compared to the CNT/TPU and GNP/TPU composites due to the improved dispersion of hybrid nanofillers. 
(a)
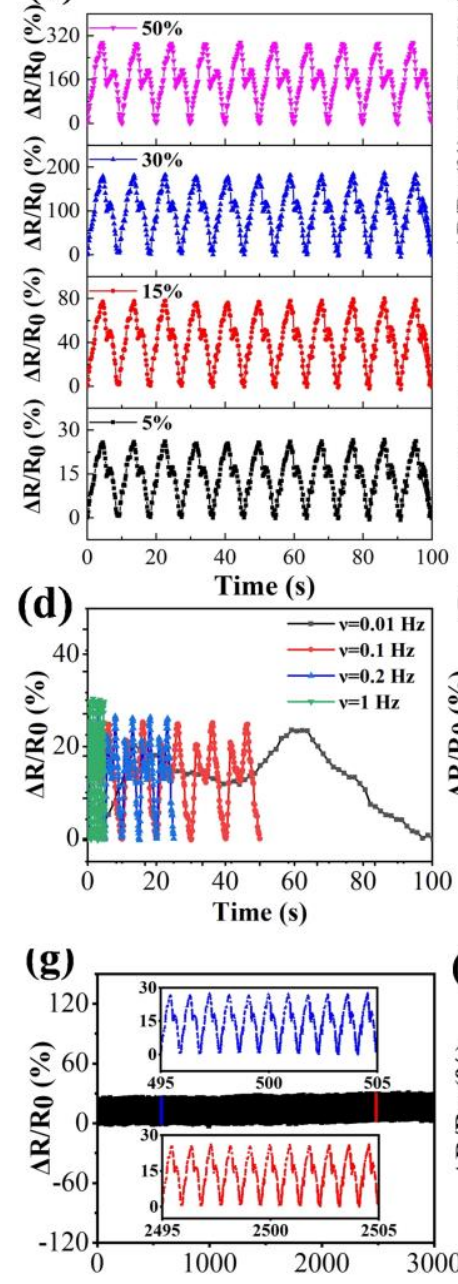

(j)

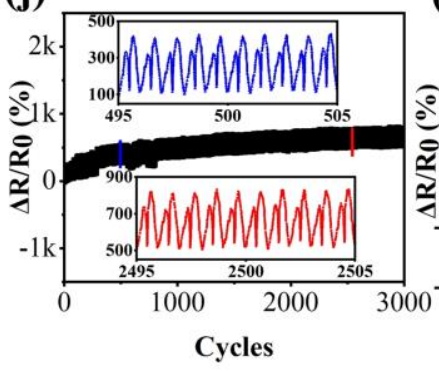

(b)

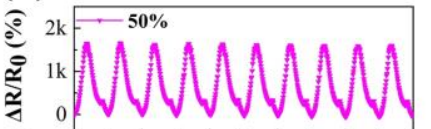

$\overbrace{}^{\circ} 1200=-30 \%$

A

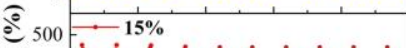

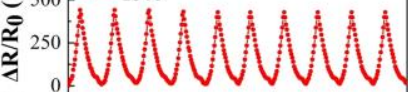

$\overbrace{200}=-5 \%$
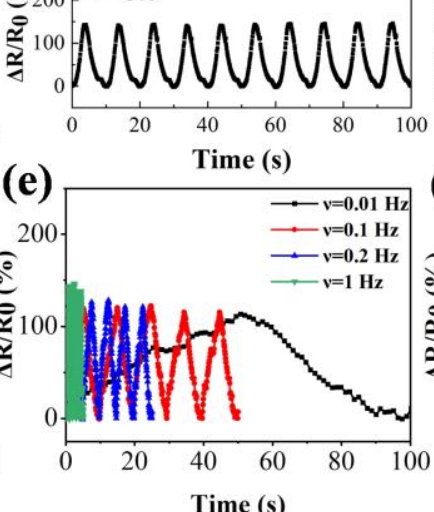

(h)

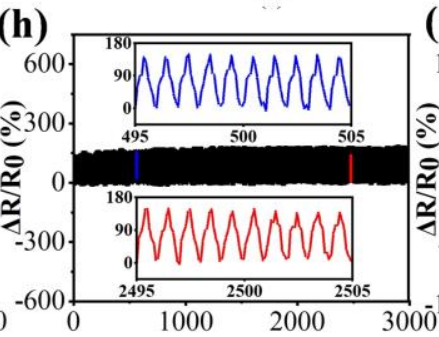

(c)
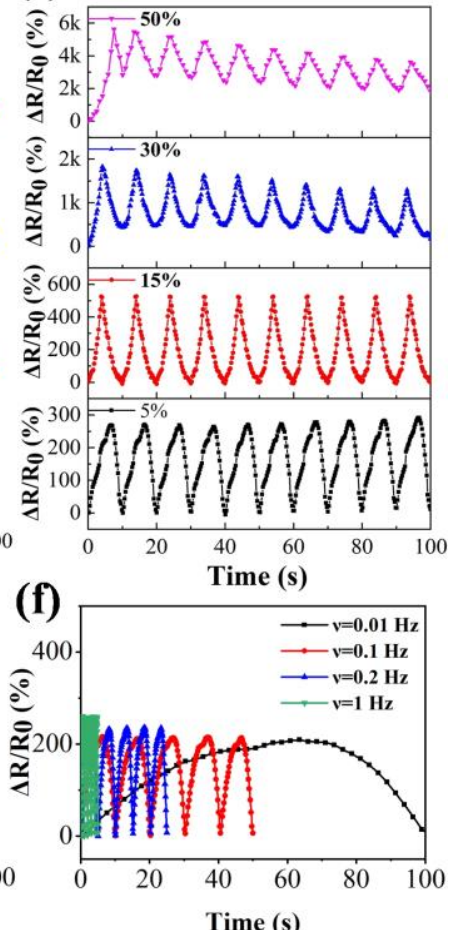

(i)

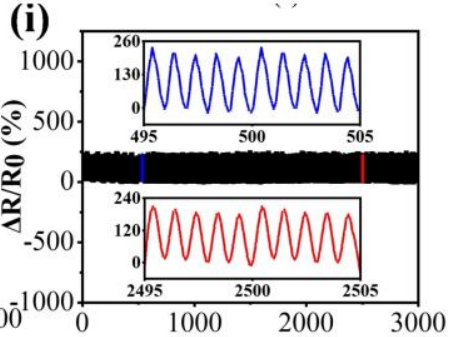

(k)

Cycles

(I)

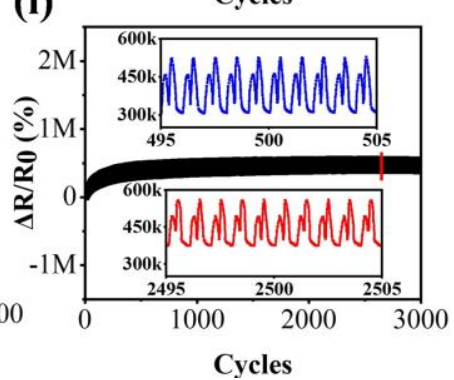

Figure 8 Variation of $\Delta R / R_{0}$ for the strain sensors with different nanofillers under cyclic stretching/releasing processes at 5, 15, 30, and 50\% strains and at a frequency of $0.1 \mathrm{~Hz}$ : (a) CNT/TPU, (b) CNT/GNP(3:1)/TPU, (c) GNP/TPU. Variation of $\Delta R / R_{0}$ for the strain sensors during cyclic loading at $5 \%$ strain and at frequencies of 0.01, 0.1, 0.2, and $1 \mathrm{~Hz}$ : (d) CNT/TPU, (e) CNT/GNP(3:1)/TPU, (f) GNP/TPU. Stability of the strain sensors up to 3000 cycles at $5 \%$ strain and at a frequency of 1 
Hz: (g) CNT/TPU, (h) CNT/GNP(3:1)/TPU, (i) GNP/TPU. Stability of the strain sensors up to 3000 cycles at $100 \%$ strain and at a frequency of $1 \mathrm{~Hz}$ : (j) CNT/TPU, (k) CNT/GNP(3:1)/TPU, (1) GNP/TPU.

\section{Modelling and mechanism}

A modelling study was conducted for the printed strain sensors to understand the strain sensing mechanism. From Fig. 9a, the total resistance $(R)$ of conductive polymer composites includes the resistance of the nanofillers $\left(R_{c n}\right)$ and the tunnel resistance between two neighboring fillers $\left(R_{t}\right)$ [30]. The total resistance $(R)$ of the nanocomposites can be calculated by Eq. (5):

$$
\begin{aligned}
& R=R_{c n}+R_{t}=\left(\frac{L}{N}\right)\left(\frac{8 \pi h d}{3 \gamma a^{2} e^{2}}\right) \exp (\gamma d) \\
& \gamma=\frac{4 \pi \sqrt{2 m \varphi}}{h}
\end{aligned}
$$

Where $N$ is the number of conductive pathways, $L$ is the number of nanofillers generating an individual conductive pathway, $a^{2}$ is the effective cross-section area, $e$ is the electron charge, $h$ is Planck's constant, $d$ is the shortest distance between the conductive nanofillers, $\varphi$ is the height of the potential barrier between nanofillers, and $m$ is the electron mass [13].

The distance between the nanofillers linearly increases from $d_{0}$ to $d$ as the nanocomposite is stretched, which improves the resistance of the nanocomposite [40]. The shortest distance between the nanofillers can be calculated by Eq. 7 .

$$
d=d_{0}\left(1+\mathrm{C}\left(\frac{\Delta l}{l_{0}}\right)\right)=d_{0}(1+C \varepsilon)
$$

where $l_{0}$ and $\Delta l$ represent the original length and deformation of the sensor, 
respectively. $\varepsilon$ is the strain, and $C$ is a constant varying with the composite systems [41].

A non-linear change in the number of conductive pathways $(N)$ under strains leads to a non-linear increase in resistance, which can be expressed as Eq. (8):

$$
N=\frac{N_{0}}{\exp \left(M \varepsilon+W \varepsilon^{2}+U \varepsilon^{3}+V \varepsilon^{4}\right)}
$$

where $M, W, U, V$ are constants, and $N_{0}$ is the number of initial conductive paths.

Eq. (9) can be given by substituting Eq. (8) and Eq. (7) into Eq. (5):

$$
R=B(1+C \varepsilon) \exp \left[A+(M+A C) \varepsilon+W \varepsilon^{2}+U \varepsilon^{3}+V \varepsilon^{4}\right]
$$

where $A=\gamma d_{0}, B=\frac{8 \pi n h d_{0}}{3 \gamma N_{0}^{2} e^{2} a^{2}}$, and $n$ is the total number of nanofillers $(n=L \times N)$.

Fig. $9 \mathrm{~b}$ illustrates the fitting curves, which are very similar to the experimental curves for the resistance of the sensor. The fitted parameters $(A, B, C, M, W, U, V)$ are shown in Table S2 (Supporting Information). Fig. 9c and d exhibit the changes of conductive pathways (change of $\mathrm{CP}, y=M x+W x^{2}+U x^{3}+V x^{4}$ ) and tunneling distance (change of TD, $y=C x$ ), respectively. In summary, the change of TD increases linearly with increasing strain. Due to the slip of GNPs, the TD and CP changes of GNP/TPU nanocomposites are more obvious. On the other hand, the TD and $\mathrm{CP}$ changes of $\mathrm{CNT} / \mathrm{TPU}$ nanocomposites are minimized due to CNTs entanglements. 
(a)
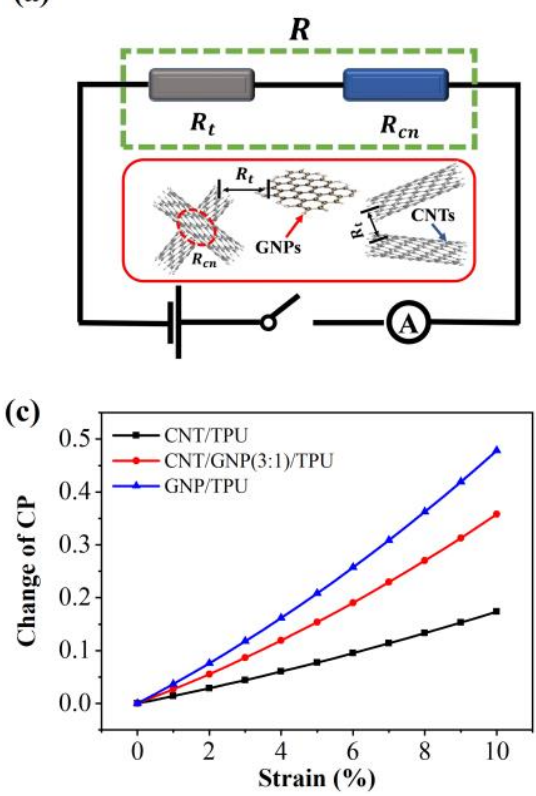

(b)

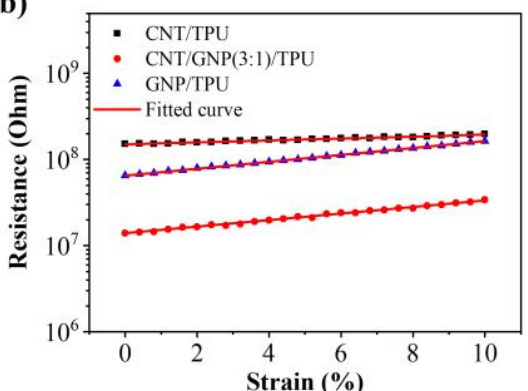

(d)

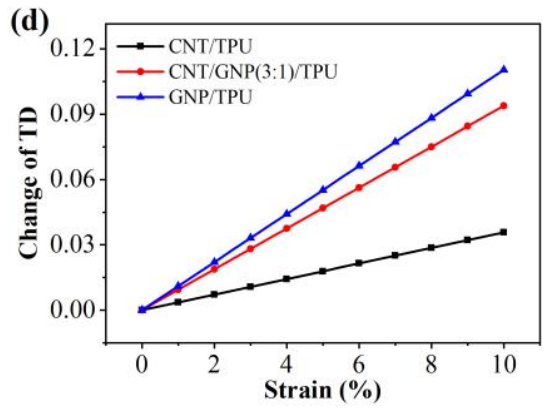

Figure 9 (a) Schematic representation of the circuit diagram of strain sensor, (b)

Experimental (dots) and theoretical (solid lines) curves for the resistance-strain relation of the printed sensors, Changes in the (c) conductive pathways and (d) tunneling distance versus strain for the strain sensors.

\section{Applications}

The high sensitivity and large detectable range of the CNT/GNP(3:1)/TPU sensor should enable it to monitor human activity, such as finger or wrist movements, facial expression changes, physiological activity, and speech recognition. Fig. 10a shows that the strain sensor can identify activities at different bending angles when fixed on the index finger. Increasing the bending angle of the finger from 0 to $90^{\circ}$, the $\Delta \mathrm{R} / \mathrm{R}_{0}$ of the sensor also increases. Fig. $10 \mathrm{~b}$ shows the signal response of the strain sensor to wrist bending. The strain sensor also has the ability to recognize the facial expressions. To investigate the ability of the printed sensor to monitor facial 
expression, the printed sensor was fixed on the upper eyelid and forehead with a bandage. As the results depicted in Fig. 10c show, the printed sensor can also be used to identify swallowing. When volunteers swallow, the sensor deforms due to muscle movements close to the esophagus, resulting in a significant change in the resistance of the sensor. From Fig. 10d, when the person blinked, the sensor responded clearly. When the facial expression recovered to the normal state, the $\Delta \mathrm{R} / \mathrm{R}_{0}$ got back to its original level. Due to the movement of abdominal muscles during breathing, strain sensors can be used to monitor the changes in the frequency of breathing, as shown in Fig. 10e. By attaching the sensor to the throat, the sensor can facilitate speech recognition by detecting the syllables of the words based on muscle movement (Fig. 10f).
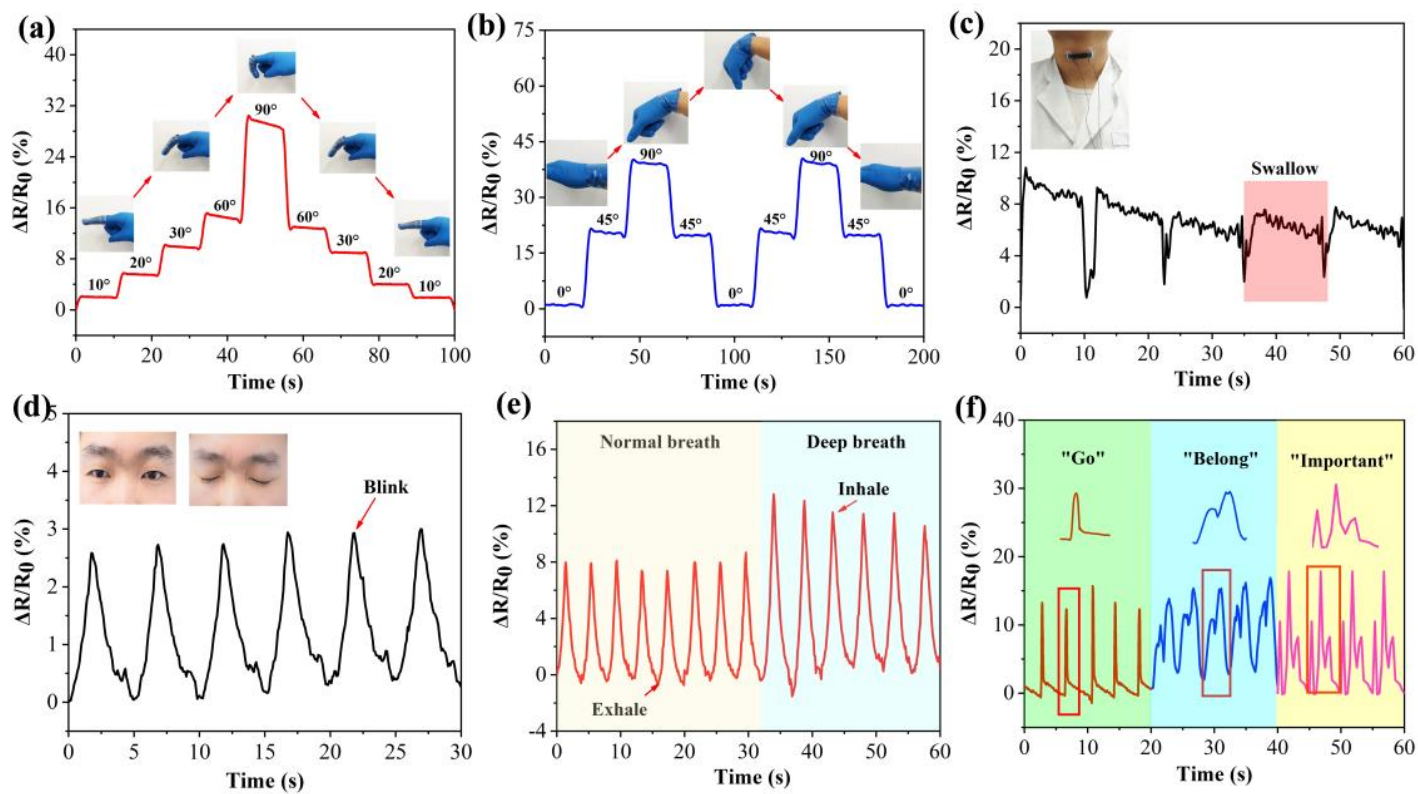

Figure 10 Strain-sensing responses of the strain sensor to repetitive (a) finger bending, (b) wrist bending, (c) swallowing, (d) blinking, (e) normal and deep breath, and (f) speaking "go", "belong”, and "important". 
In this work, highly flexible strain sensors based on CNT/TPU, GNP/TPU, and CNT/GNP/TPU nanocomposites were fabricated by FFF 3D printing. The dispersion, printability, as well as the electrical, tensile, and sensing properties of the printed composites were systematically investigated. Due to the synergistic effect of CNTs and GNPs, the improved dispersion of CNTs in the TPU matrix was obtained, and the electrical and tensile properties of the 3D printed sensor were significantly enhanced. Compared with the GNP/TPU and CNT/TPU composite filaments, CNT/GNP(3:1)/TPU filaments exhibited outstanding elastic modulus and critical buckling pressure of (21.2 MPa and $8.2 \mathrm{kPa}$, respectively) at a total nanofiller content of $2 \mathrm{wt} \%$. In addition, the CNT/GNP(3:1)/TPU composite showed higher strength (12.6 MPa) and tensile modulus (23.2 MPa), and comparative elongation (540.3\%). The introduction of GNPs to the matrix generated a more complete conductive network in the final composite, which effectively reduced the percolation threshold from 1.98 to $1.42 \mathrm{wt} \%$. Furthermore, the printed CNT/GNP(3:1)/TPU sensor exhibited an outstanding sensitivity ( $\mathrm{GF}=136327.4$ at $250 \%$ strain), a large workable strain range (up to 250\%), and good stability (3000 cycles). To analyze the mechanism of strain sensing, modelling based on tunnel theory was performed. A good agreement between the theoretical and experimental results was found. The ability of strain sensors to monitor limb motions, physiological activities, and speech recognition has also been demonstrated. This work offers an effective method for the 
3D printing of high-performance flexible strain sensors with potential applications in human-computer interaction, smart wearable devices, and medical monitoring equipment.

\section{Acknowledgements}

This work was supported by the Sichuan Science and Technology Program (2017HH0086, 2017JY0152), Sichuan Provincial University Key Laboratory of Oil and Gas Field Materials (No. X151519KCL05), and the Scientific Research Foundation for the Returned Overseas Chinese Scholars of Sichuan Province.

\section{References}

[1] Zhao K, Niu W, Zhang S (2020) Highly stretchable, breathable and negative resistance variation textile strain sensor with excellent mechanical stability for wearable electronics. J Mater Sci 2020;55:2439-53. doi:10.1007/s10853-019-04189-x.

[2] Hwang B, Choi S, Lee K, Kim J (2018) Highly stretchable and transparent electrode fi lm based on SWCNT / Silver nanowire hybrid nanocomposite. Compos Part B 2018;151:1-7. doi:10.1016/j.compositesb.2018.06.004.

[3] Meng Q, Liu Z, Han S, Xu L(2019) I F E S sciences for life A facile approach to fabricate highly sensitive, flexible strain sensor based on elastomeric / graphene platelet composite film. J Mater Sci 2019;54:10856-70. doi:10.1007/s10853-019-03650-1.

[4] Dou Y, Liu H, Peng J, et al (2016) A green method for preparation of $\mathrm{CNT/CS/AgNP} \mathrm{composites} \mathrm{and} \mathrm{evaluation} \mathrm{of} \mathrm{their} \mathrm{catalytic} \mathrm{performance.} \mathrm{J}$ Mater Sci 2016;51:5685-94. doi:10.1007/s10853-016-9871-1.

[5] Zhang R, Deng H, Valenca R, et al (2013) Strain sensing behaviour of elastomeric composite films containing carbon nanotubes under cyclic loading. Compos Sci Technol 2013;74:1-5. doi:10.1016/j.compscitech.2012.09.016.

[6] Wang Y, Hao J, Huang Z, et al (2018) Flexible electrically resistive-type strain sensors based on reduced graphene oxide-decorated electrospun polymer fibrous mats for human motion monitoring. Carbon 2018;126:360-71. doi:10.1016/j.carbon.2017.10.034. 
[7] Kim JY, Ji S, Jung S, et al (2017) 3D printable composite dough for stretchable, ultrasensitive and body-patchable strain sensors. Nanoscale 2017;9:11035-46. doi:10.1039/c7nr01865g.

[8] Liu H, Gao J, Huang W, et al (2016) Electrically conductive strain sensing polyurethane nanocomposites with synergistic carbon nanotubes and graphene bifillers. Nanoscale 2016;8:12977-89. doi:10.1039/c6nr02216b.

[9] Ma Z, Wei A, Ma J, Shao L, et al (2018) Lightweight, compressible and electrically conductive polyurethane sponges coated with synergistic multiwalled carbon nanotubes and graphene for piezoresistive sensors. Nanoscale 2018;10:7116-26. doi:10.1039/c8nr00004b.

[10] Peng X, Wu K, Hu Y, et al (2018) A mechanically strong and sensitive $\mathrm{CNT} / \mathrm{rGO}-\mathrm{CNF}$ carbon aerogel for piezoresistive sensors. J Mater Chem A 2018;6:23550-9. doi:10.1039/C8TA09322A.

[11] Zhao X, Xu L, Chen Q, et al (2019) Highly Conductive Multifunctional rGO/CNT Hybrid Sponge for Electromagnetic Wave Shielding and Strain Sensor. Adv Mater Technol 2019;4:1900443. doi:10.1002/admt.201900443.

[12] Ligon SC, Liska R, Stampfl J, et al (2017) Polymers for 3D Printing and Customized Additive Manufacturing. Chem Rev 2017;117:10212-90. doi:10.1021/acs.chemrev.7b00074.

[13] Kim DW, Lim JH, Yu J (2019) Efficient prediction of the electrical conductivity and percolation threshold of nanocomposite containing spherical particles with three-dimensional random representative volume elements by random filler removal. Compos Part B Eng 2019;168:387-97. doi:10.1016/j.compositesb.2019.03.038.

[14] Xiang D, Zhang X, Li Y, et al (2019) Enhanced performance of 3D printed highly elastic strain sensors of carbon nanotube / thermoplastic polyurethane nanocomposites via non-covalent interactions. Compos Part B Eng 2019;176. doi:10.1016/j.compositesb.2019.107250.

[15] Mu Q, Wang L, Dunn CK, et al (2017) Digital light processing 3D printing of conductive complex structures. Addit Manuf 2017;18:74-83. doi:10.1016/j.addma.2017.08.011.

[16] Li Z, Wang Z, Gan X, et al (2017) Selective Laser Sintering 3D Printing: A Way to Construct 3D Electrically Conductive Segregated Network in Polymer Matrix. Macromol Mater Eng 2017;302:1-10. doi:10.1002/mame.201700211.

[17] Odent J, Wallin TJ, Pan W, et al (2017) Highly Elastic, Transparent, and Conductive 3D-Printed Ionic Composite Hydrogels. Adv Funct Mater 2017;27:1-10. doi:10.1002/adfm.201701807.

[18] Christ JF, Aliheidari N, Ameli A, Pötschke P (2017) 3D printed highly elastic strain sensors of multiwalled carbon nanotube/thermoplastic polyurethane nanocomposites. Mater Des 2017;131:394-401. doi:10.1016/j.matdes.2017.06.011.

[19] Huang P, Xia Z, Cui S (2017) 3D printing of carbon fiber-filled conductive silicon rubber. Mater Des 2018;142:11-21. doi:10.1016/j.matdes.2017.12.051.

[20] Xiang D, Zhang X, Harkin-Jones E, et al (2020) Synergistic effects of hybrid 
conductive nanofillers on the performance of 3D printed highly elastic strain sensors. Compos Part A Appl Sci Manuf 129:105730. https://doi.org/10.1016/j.compositesa.2019.105730

[21] Deng H, Lin L, Ji M, et al (2013) Progress on the morphological control of conductive network in conductive polymer composites and the use as electroactive multifunctional materials. Prog Polym Sci 2014;39:627-55. doi:10.1016/j.progpolymsci.2013.07.007.

[22] Xiang D, Wang L, Tang Y, et al (2018) Damage self-sensing behavior of carbon nanofiller reinforced polymer composites with different conductive network structures. Polymer 2018;158:308-19. doi:10.1016/j.polymer.2018.11.007.

[23] Christ JF, Aliheidari N, Ameli A, Pötschke P (2017) 3D printed highly elastic strain sensors of multiwalled carbon nanotube/thermoplastic polyurethane nanocomposites. Mater Des 2017;131:394-401. doi:10.1016/j.matdes.2017.06.011.

[24] Li Y, Zhou B, Zheng G, et al (2018) Continuously prepared highly conductive and stretchable SWNT/MWNT synergistically composited electrospun thermoplastic polyurethane yarns for wearable sensing. J Mater Chem C 2018;6:2258-69. doi:10.1039/c7tc04959e.

[25] Zhao H, Bai J (2015) Highly sensitive piezo-resistive graphite nanoplatelet-carbon nanotube hybrids/polydimethylsilicone composites with improved conductive network construction. ACS Appl Mater Interfaces 2015;7:9652-9. doi:10.1021/acsami.5b01413.

[26] Lachhab A, Robin E, Le Cam JB, et al (2017) Thermomechanical analysis of polymeric foams subjected to cyclic loading: Anelasticity, self-heating and strain-induced crystallization. Polymer2017;126:19-28. doi:10.1016/j.polymer.2017.08.010.

[27] Reulier M, Matadi Boumbimba R, Walsh Korb Z, Vaudemont R, Avérous L (2017) Thermomechanical and cyclic behavior of biocomposites based on renewable thermoplastics from dimer fatty acids. J Appl Polym Sci 2017;134:1-13. doi:10.1002/app.44610.

[28] Roh E, Hwang BU, Kim D, et al (2015) Stretchable, Transparent, Ultrasensitive, and Patchable Strain Sensor for Human-Machine Interfaces Comprising a Nanohybrid of Carbon Nanotubes and Conductive Elastomers. ACS Nano 2015;9:6252-61. doi:10.1021/acsnano.5b01613.

[29] Wang L, Xiang D, Harkin-jones E,et al (2019) A Flexible and Multipurpose Piezoresistive Strain Sensor Based on Carbonized Phenol Formaldehyde Foam for Human Motion Monitoring 2019;1900492:1-9. doi:10.1002/mame.201900492.

[30] Chen Q, Xiang D, Wang L, et al (2018) Facile fabrication and performance of robust polymer/carbon nanotube coated spandex fibers for strain sensing. Compos Part A Appl Sci Manuf 2018;112:186-196. doi:10.1016/j.compositesa.2018.06.009.

[31] Liao X, Liao Q, Yan X, et al (2015) Flexible and highly sensitive strain sensors 
fabricated by pencil drawn for wearable monitor. Adv Funct Mater 2015;25:2395-401. doi:10.1002/adfm.201500094.

[32] Zeng Z, Seyed Shahabadi SI, Che B, et al (2017) Highly stretchable, sensitive strain sensors with a wide linear sensing region based on compressed anisotropic graphene foam/polymer nanocomposites. Nanoscale 2017;9:17396404. doi:10.1039/c7nr05106a.

[33] Amjadi M, Yoon YJ, Park I (2015) Ultra-stretchable and skin-mountable strain sensors using carbon nanotubes-Ecoflex nanocomposites. Nanotechnology 2015;26:375501. doi:10.1088/0957-4484/26/37/375501.

[34] Liang B, Lin Z, Chen W, et al (2018) Ultra-stretchable and highly sensitive strain sensor based on gradient structure carbon nanotubes. Nanoscale 2018;10:13599-606. doi:10.1039/c8nr02528b.

[35] Lin Y, Liu S, Chen S, Wei Y, et al (2016) A highly stretchable and sensitive strain sensor based on graphene-elastomer composites with a novel double-interconnected network. J Mater Chem C 2016;4:6345-52. doi:10.1039/c6tc01925k.

[36] Zhong W, Liu C, Xiang C, et al (2017) Continuously Producible Ultrasensitive Wearable Strain Sensor Assembled with Three-Dimensional Interpenetrating Ag Nanowires/Polyolefin Elastomer Nanofibrous Composite Yarn. ACS Appl Mater Interfaces 2017;9:42058-66. doi:10.1021/acsami.7b11431.

[37] He Z, Zhou G, Byun JH, et al (2019) Highly stretchable multi-walled carbon nanotube/thermoplastic polyurethane composite fibers for ultrasensitive, wearable strain sensors. Nanoscale 2019;11:5884-90. doi:10.1039/C9NR01005J.

[38] Chen S, Wu R, Li P, et al (2018) Acid-Interface Engineering of Carbon Nanotube/Elastomers with Enhanced Sensitivity for Stretchable Strain Sensors. ACS Appl Mater Interfaces 2018;10:37760-6. doi:10.1021/acsami.8b16591.

[39] Zhou X, Zhu L, Fan L, et al (2018) Fabrication of Highly Stretchable, Washable, Wearable, Water-Repellent Strain Sensors with Multi-Stimuli Sensing Ability. ACS Appl Mater Interfaces 2018;10:31655-63. doi:10.1021/acsami.8b11766.

[40] Lozano-Pérez C, Cauich-Rodríguez J V., Avilés F (2016) Influence of rigid segment and carbon nanotube concentration on the cyclic piezoresistive and hysteretic behavior of multiwall carbon nanotube/segmented polyurethane composites. Compos Sci Technol 2016;128:25-32. doi:10.1016/j.compscitech.2016.03.010.

[41] Lin L, Liu S, Zhang Q, et al (2013) Towards tunable sensitivity of electrical property to strain for conductive polymer composites based on thermoplastic elastomer. ACS Appl Mater Interfaces 2013;5:5815-5824. doi:10.1021/am401402x. 
Click here to access/download Supplementary Material Supporting information -2020-7-6.docx 